\title{
Functional evaluation of iodoacetic acid induced photoreceptor degeneration in the cat
}

\author{
NAN Yan ${ }^{1 \dagger}$, ZHANG Qin $^{2 \dagger}$, REN ChaoRan ${ }^{1 \dagger}$, HUANG Xin ${ }^{1}$, GAO Jie ${ }^{1}$, \\ LI XiaoXin $^{2^{*}} \&$ PU MingLiang ${ }^{1^{*}}$ \\ ${ }^{1}$ Department of Anatomy/Embryology, School of Basic Medical Sciences, Peking University, Beijing 100191, China; \\ ${ }^{2}$ Department of Ophthalmology, People's Hospital, Peking University, Beijing 100044, China
}

Received March 20, 2013; accepted April 10, 2013; published online May 7, 2013

\begin{abstract}
Iodoacetic acid (IAA) has been applied to different species to acutely induce photoreceptor degeneration. The purpose of the present study was to use this toxin to thoroughly eliminate photoreceptors and induce complete blindness in the cat. IAA was delivered by single ear vein injection $\left(20 \mathrm{mg} \mathrm{kg}^{-1}\right)$. Six months after the IAA treatment, functional evaluations including pupillary light reflex (PLR), electroretinogram (ERG), visual behavior tests were performed. Morphological examinations were carried out after the functional evaluation. The present result shows that, six months after the IAA application, animals lost visual functions and became completely blind. High dose IAA application via ear vein delivery created an acute and reliable complete photoreceptor degeneration model in the cat. This model can be applied to genetic and cellular therapies for visual function restoration.
\end{abstract}

iodoacetic acid (IAA), photoreceptor, retinal degeneration, cat

Citation: $\quad$ Nan Y, Zhang Q, Ren C R, et al. Functional evaluation of iodoacetic acid induced photoreceptor degeneration in the cat. Sci China Life Sci, 2013, 56: 524-530, doi: 10.1007/s11427-013-4483-3

Iodoacetic acid (IAA) inhibits anaerobic glycolysis by reacting with the sulfhydryl group of glyceraldehyde-3phosphate dehydrogenase [1], resulting in lower production of metabolic energy that causes impaired visual function and photoreceptor death. IAA has been applied to different species to induce photoreceptor degeneration including rat [2], rabbit [3-8], Ground squirrel [9], cat and monkey [4]. However, at the same time the inner retina and central visual system are well preserved, which is a similar case that could be found in retinitis pigmentosa (RP) and age related macular degenerations (AMD) of human. By intravenous delivery of IAA via ear vein, it has been demonstrated that IAA could quickly produce a massive photoreceptor depletion in large mammals such as swine and results in severe

$\dagger$ Contributed equally to this work

*Corresponding author (email: dr_lixiaoxin@163.com; mpu@hsc.pku.edu.cn) visual impairment whereas retinal ganglion cells are spared $[10,11]$.

IAA induced retinal degeneration has been widely applied to various retinal disease animal models. Depending on the purpose of the experiments, the most of current applications are interested in inducing partial photoreceptor or pigment epithelium degeneration and create a certain retinal disease of given stage $[2,3,7,10,12-14]$. For photoreceptor degeneration, it is reported that after the treatment, rods were completely eliminated while cones were less affected [4]. Similar results were observed in rat [2]. Interestingly, the long lasting damage to the photoreceptors did not lead to a reorganization of the surviving cells, at least with survival as long as six months [7]. Nevertheless, the degree of IAA induced injury varies with the dosage and the same dosage of IAA induced photoreceptor degeneration varies among individual animals, occasionally different level of injury 
observed between eyes of the same animal $[7,10,14]$. These differences clearly make it challenging to determine the extent of damage by IAA. To date, there is no large mammal animal model available for the complete blindness that mimics the end stage retinal disease such as RP and AMD. We sought to develop an animal model of complete blindness induced by intravenous injection of IAA in the feline.

Here we report a newly developed feline model of acute and complete photoreceptor degeneration with only one intravenous injection of IAA. Six months after the IAA treatment a series of physiological, behavioral, and morphological examinations were performed, and IAA treated animal demonstrated complete lost visual functions. Together, this model could offer an indispensable tool to evaluate the efficacy of various treatments for visual function restore.

\section{Methods}

\subsection{Animals}

Young adult cats (2-3 years old) of either sex were used in this experiment. Cats were kept at $12 \mathrm{~h} / 12 \mathrm{~h}$ light and dark environment. Food and water were provided ad libitum. All experiments were performed in accordance with Peking University guidelines for animal research and the ARVO Statement for the Use of Animals in Ophthalmic and Vision Research. The experimental animal protocol used in this study was approved by Peking University Institutional Animal Care and Use Committee (IACUC). Cats received an intravenous injection of $20 \mathrm{mg} \mathrm{kg}^{-1}$ body weight of sterile (PH balanced) IAA (Sigma Chemical Corp., St Louis, MO, USA).

\subsection{Pupillary light reflex (PLR)}

PLR measurements of pupillary area were made as a function of retinal illuminance on 11 adult cats, five from normal control and the rest from IAA treated cats. The cats were not treated with any anesthetics, anticholinergic, or antispasmodic agents that could dilate pupillary size such as atropine. Pupillary area was photographed while cats binocularly viewed a flash light source that had at $30 \mathrm{~lx}$ first then $1200 \mathrm{~lx}$, respectively. Thus, the shooting distance varied between the camera and a moving animal. Consequently, the pictured pupil size varied from animal to animal even if the photographs were taken under the same luminance levels. To compensate for this error and estimate the captured pupillary size objectively, we calculated the relative ratio of pupillary area vs. the entire corneal area. This ratio properly reflects the pupillary area variation under different intensities of light illumination. All measurements were made independently by at least two investigators and checked for consistency. To avoid circadian effects on pupillary areas, animals were housed at $12 \mathrm{~h} / 12 \mathrm{~h}$ light and dark lighting environment. All measurements were performed between
9:00 and 10:00 a.m.. Only animals where PLRs were reliably recorded through the entire protocol were included. Due to technical difficulties in the measurement, the failed recordings were not included. Images captured were processed with a graphic software (Photoshop CS5, Adobe Corp., San Jose, California, USA).

\subsection{Electroretinogram (ERG)}

The full-field (Ganzfeld) ERG was recorded with an electroretinography recording system (RETIport, Roland Consult, Brandenburg, Germany). Recordings were performed following the ISCEV standard for full-field clinical electroretinography. The ERG recordings were conducted in three normal and six IAA treated animals. All animals were assessed with the full-field ERG. Cats were anesthetized using the protocol previously described by Nan et al. [15]. During the recording, the animal body temperature was maintained at $37^{\circ} \mathrm{C}$ with a heating blanket. The eyes were anesthetized with $1 \%$ tetracaine hydrochloride eye drops. The pupil was dilated with topical application of eye drops containing $0.5 \%$ tropicamide and $0.5 \%$ phenylephrine (Santen, Japan). Animals were dark adapted for 30 min before the scotopic ERG recording. The ERG was recorded with an active corneal contact lens electrode (ERG-Jet F-06 Lens, Fabrinalsa, Sweden). The reference and ground electrodes were inserted subcutaneously at the forehead skin and the tail, respectively. The cornea was kept moist with $0.1 \%$ Sodium Hyaluronate eye drops (Santen, Japan). The intensity of white light stimulation for scotopic ERG was set at $0.01 \mathrm{~cd} \mathrm{~m}^{-2}$ first. The intensity was then increased to $3 \mathrm{~cd} \mathrm{~m}^{-2}$. The oscillatory potentials were recorded with the same intensity $\left(3 \mathrm{~cd} \mathrm{~m}^{-2}\right)$. The cat was then light adapted in a photopic background $\left(25 \mathrm{~cd} \mathrm{~m}^{-2}\right)$ for $30 \mathrm{~min}$. The stimulus intensity for photopic ERG was $3 \mathrm{~cd} \mathrm{~m}^{-2}$. Each recording was averaged three times at the above luminance levels. The $30 \mathrm{~Hz}$ flicker stimulation was recorded at the same intensity but averaged eight times.

\subsection{Behavioral evaluation}

Three normal and six IAA-treated cats that had no PLR and ERG responses were selected for behavioral evaluation under day light conditions. The testing course was $15 \mathrm{~m}$ long and $1.5 \mathrm{~m}$ wide. There were eight barricades in the course. The distance between the two barricades was $2 \mathrm{~m}$. Each barricade was $1.2 \mathrm{~m}$ wide and was placed on the one side wall of the course alternatively to form a Z-shaped obstacle path. This would allow a $0.8 \mathrm{~m}$ space through which cats could navigate between the barricade and side wall. Each barricade had the option to slide to the left or right side wall of the course. The barricade positions were changed randomly between runs to account for learning behavior. Each animal was allowed to run three times. During a test, a cat was released at the entrance of the course; the transit time 
was measured from the first forward motion to passing through the exit. The time course of animals negotiating through the obstacle path and the number of collisions made by the cat while negotiating the path were recorded with a video camera (Powershot 870, Canon USA, New York).

\subsection{Histological examination}

After the behavioral tests, four cats were sacrificed, two of them were normal and the rest were six months after IAA treatment, respectively. Both of the IAA-treated cats have no pupillary light reflex and ERG responses. The details of immunohistochemical staining have been described elsewhere [15]. Briefly, eyes were enucleated while animals were under deep anesthesia and the lens and vitreous were immediately removed with a pair of fine-tip forceps. Then eyecups were fixed by a $4 \%$ paraformaldehyde in phosphate buffer $\left(0.1 \mathrm{~mol} \mathrm{~L}^{-1}, \mathrm{pH} 7.4\right)$ for $90 \mathrm{~min}$ at room temperature. After gradient elution using 10\%, 20\%, 30\% sucrose $(\mathrm{pH}$ 7.4), OCT embedded retinas were froze and cut at a thickness of $10 \mu \mathrm{m}$. The cryosections were mounted on glass slides and allowed to dry overnight. For histochemical examination, sections were stained using a standard hematoxylin-eosin-saffranin staining protocol and viewed with a Olympus microscope (Xi-71, Olympus Corp., Japan). Images of stained retinas were captured with a CCD camera (700D; Nikon, Tokyo, Japan) coupled to a desktop computer equipped with Photoshop graphic software (CS5 Photoshop, Adobe Corp., San Jose, California, USA).

\subsection{Statistical analysis}

Results are reported as mean \pm SEM. ANOVA and Student's $t$-test were used for the determination of statistical significance among treatment groups.

\section{Results}

IAA induced photoreceptor degeneration resulted temporary loss visual function during the first week in all treated animals. However, some cats gradually regained visual function during next couple of months or so but others did not show any sign of visual guided behavior or visual function. Thus we suspect the latter became permanently blind. To objectively determine if these animals have any residual visual function we performed the following tests six months after the IAA treatment.

\subsection{PLR and relative ratio of pupillary area}

Animals were treated with IAA $\left(20 \mathrm{mg} \mathrm{kg}^{-1}\right)$ by intravenous injection at an ear vein that is running along the outer edge towards the tip of the ear. Functional evaluations were performed six months post IAA treatment. Controls were age-matched. Figure 1 compares representative PLR responses between a control (Figure 1B and D) and an IAA treated cat (Figure 1A and $\mathrm{C}$ ) six months post-injection. Notice Figure 1C, due to the shooting angle of the camera, the widely dilated left pupil was captured. Compared to a weak light illumination (30 lx, Figure 1D), the pupillary area of control animal was reduced in response to a strong flash light illumination (1500 lx, Figure 1B), whereas that of IAA treated animal showed a less constricted pupillary area (Figure 1A and C). To quantitatively evaluate the pupillary area under these testing conditions, we repeated the measurement with normal and IAA treated animals (normal: $n=5,10$ eyes; IAA: $n=4,8$ eyes). As shown in Figure 2, it reveals the ratio of relative pupillary area changes before and after light stimulation between the control and IAA

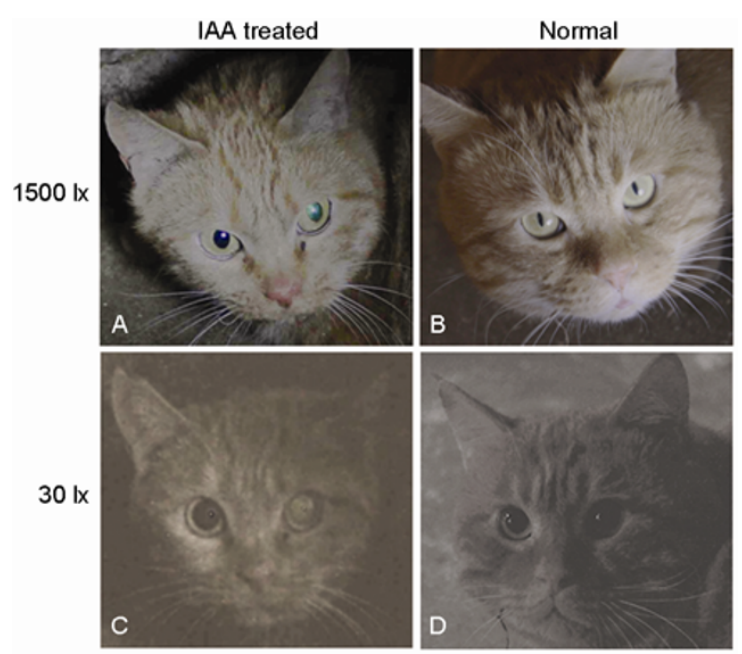

Figure 1 Photographs of pupillary light reflex (PLR) under different illuminance levels in normal and IAA treated cats. A, IAA treated cat PLR under 1500 lx light exposure. B, Normal cat PLR under 1500 lx. C, PLR of an IAA treated cat under 30 lx illuminance. D, Normal cat PLR under 30 lx.

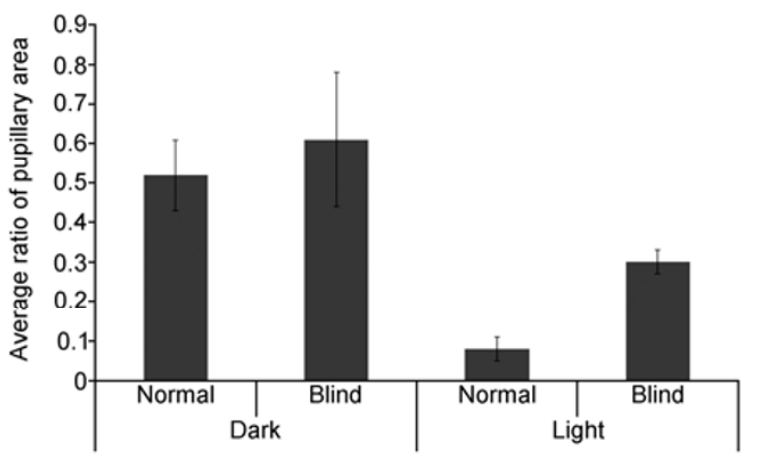

Figure 2 Histogram comparing averaged pupillary area under light and dark conditions in normal and IAA treated cats. Average ratio of pupillary area was taken under dark (30 lx) first, then light illumination (1500 lx) (mean \pm STDEV). Under $30 \mathrm{~lx}$ illumination, the averaged ratio of pupillary area was $0.52 \pm 0.09$ and $0.61 \pm 0.17$ for controls and IAA treated animals, respectively; under $1500 \mathrm{~lx}$, it was $0.08 \pm 0.03$ and $0.3 \pm 0.11$ for controls and IAA treated animals, respectively. Vertical coordinate shows the averaged ratio of pupillary area and horizontal axis includes normal and IAA treated animals tested under two illuminance levels $(P<0.01$, respectively, ANOVA). 
treated animals (mean \pm STDEV). The averaged ratio under $30 \mathrm{~lx}$ was $0.52 \pm 0.09$ and $0.61 \pm 0.17$ for controls $(n=5)$ and IAA treated animals $(n=4)$, respectively. The ratio decreased under 1500 lx illumination (controls: 0.08 $\pm 0.03, n=5$; IAA treated: $0.3 \pm 0.03, n=4)$. As show in Figure 2, these changes were statistically significant for both controls and IAA treated cats ( $t$ test: normal, $P<0.0001$; IAA, $P<0.0007)$. However, as the comparison was made between the ratios of changes in averaged pupillary areas under the two illuminance levels, the magnitude of pupillary area change was $6.99 \pm 2.59$ and $2.15 \pm 0.35(P<0.01, t$-test $)$, for controls and IAA treated animals, respectively.

\subsection{Full-field electroretinogram (ff-ERG)}

Since the reduced PLR response only reflects the animal's light reflex but not retinal visual function. Therefore, we performed ERG screen. Figure 3 compares representative dark- and light-adapted ff-ERG responses between normal and IAA treated animals. Figure $3 \mathrm{~A}$ plots the mean a(baseline to trough) and b-wave (baseline to peak) responses for control (upper left chart) and IAA-treated cats (upper right chart) at the stimulus intensity of $0.01 \mathrm{~cd} \mathrm{~m}^{-2}$. As shown in the panel, the ff-ERG responses of a normal and the IAA-treated cat exhibited completely different response patterns. In comparison with the former, the ff-ERG response of the latter was completely lost. Figure $3 \mathrm{~B}$ shows their ERG responses to visual stimulation intensity of $3 \mathrm{~cd} \mathrm{~m}^{-2}$. Figure
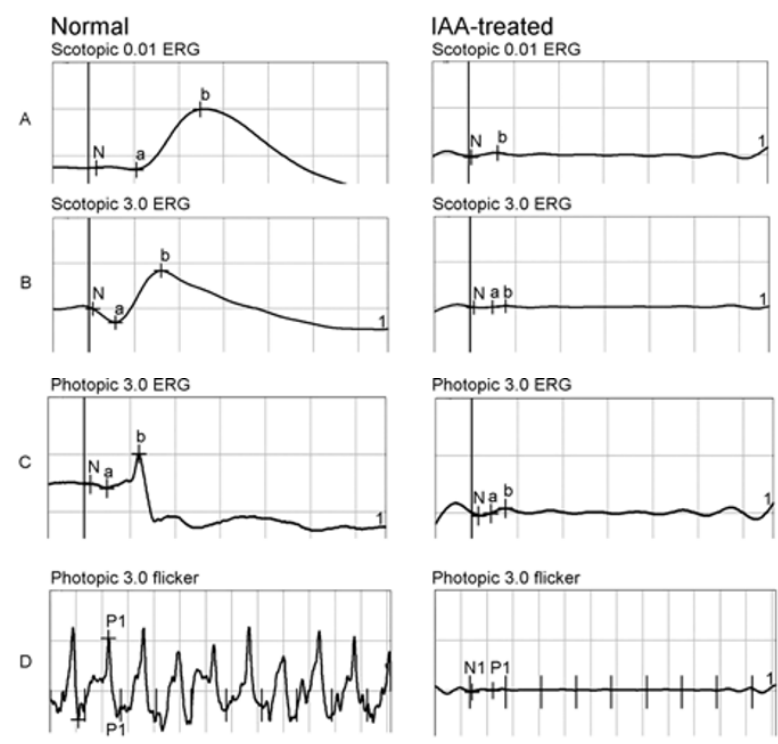

Figure 3 Full-field electroretinogram of normal and IAA treated animals. Left column, normal cat; right column, IAA treated cat. A, Scotopic 0.01: the animal was dark adapted for $30 \mathrm{~min}$, the intensity of light stimulation was $0.01 \mathrm{~cd} \mathrm{~m}^{-2}$, and the vertical coordinate was response amplitude, 250 $\mu \mathrm{V} \mathrm{div}{ }^{-1}$. The abscissa is time scale $\left(25 \mathrm{~ms} \mathrm{div}^{-1}\right)$. a, a-wave; b, b-wave. B, Scotopic 3 ERG: the intensity of light stimulus increased to $3 \mathrm{~cd} \mathrm{~m}^{-2}$, the amplitude is $500 \mu \mathrm{V} \mathrm{div}{ }^{-1}$. C, Photopic 3 ERG: oscillatory potential 100 $\mu \mathrm{V} \mathrm{div}{ }^{-1}$. D, Photopic 3 flicker: the animal was light adapted at $25 \mathrm{~cd} \mathrm{~m}^{-2}$ for $30 \mathrm{~min}$ before the visual stimulation, response amplitude $50 \mu \mathrm{V} \mathrm{div}{ }^{-1}$.
$3 \mathrm{C}$ depicts the oscillatory potentials in response to stimulation intensity of $3 \mathrm{~cd} \mathrm{~m}^{-2}$. Figure 3D compares flicker ERG responses of normal and IAA treated animals. Together, ERG response profiles under scotopic and photopic stimulation patterns demonstrate that both rod and cone driven inputs are completely extinguished after the IAA treatment. To quantitatively assess the impact of IAA, the measurements were conducted in more control $(n=3)$ and IAA treated animals $(n=6)$. As shown in Figure 4, ERG a-, b-wave components and flicker responses were drastically reduced in IAA treated animals. Thus, the present electrophysiological evidence indicates that IAA treatment abolished both rods and cones functions.

\subsection{Vision behavioral test}

The ERG result suggests that IAA treatment caused substantial loss of $a$ and $b$ waves and scotopic and photopic retinal functions were substantially eliminated. However, it remained to be verified if the IAA treated animal had any residual visual functions. To access visual function of IAA treated animals, we conducted a visual behavioral test in an obstacle-avoidance course. Three normal and six IAA treated cats were selected for the test. During the test animals were placed at one end of the testing course (width, $1.5 \mathrm{~m}$; length, $15 \mathrm{~m}$ ), the animal had to avoid obstacles and run through the course. The time course of animals moving through the obstacle path and the number of barricades crashed were recorded. As shown in video clips, compared to normal cat (clip A), the IAA treated animal showed a completely different running pattern that showed no indication of using visual organ (clip B). We quantitatively analyzed this visual behavior pattern, as shown in Figure 5. In comparison with controls $(7.21 \pm 2.55, n=5)$, IAA treated animals spent a significantly long time in the course

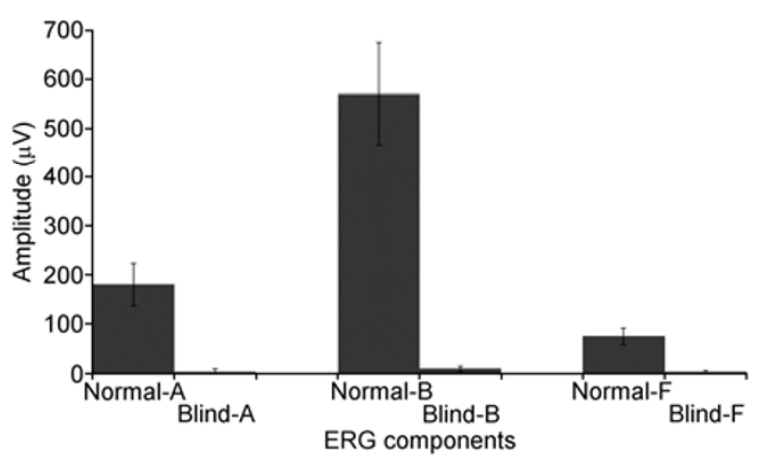

Figure 4 Histogram comparing averaged amplitudes of D-ERG responses between normal and IAA treated cats. Columns Normal-A and Blind-A, comparison of the averaged a-wave between normal $(180.67 \pm 43.30, n=3)$ and IAA treated cats $(3.81 \pm 4.77, n=6)$. Columns Normal-B and Blind-B, comparison of the averaged b-wave between normal $(571.17 \pm 104.88, n=3)$ and IAA treated cats $(9.45 \pm 5.72, n=6)$. Column normal-F and Blind-F, comparison of flicker ERG between normal $(74.9 \pm 16.84, n=3)$ and IAA-treated animals $(3.4 \pm 2.96, n=6)$. The vertical coordinate depicts the averaged amplitudes $(\mu \mathrm{V})$ and the horizontal axis shows ERG wave components. Conventions are as for Figure 3 ( $P<0.01$, respectively, ANOVA). 


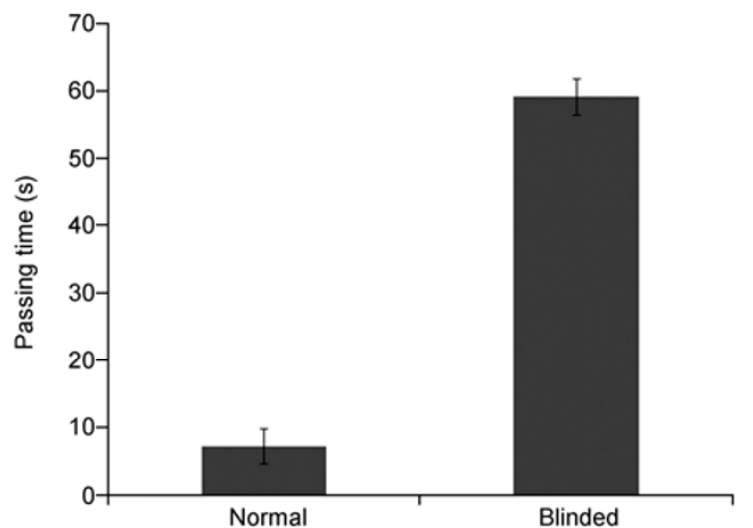

Figure 5 Averaged passing times in normal and IAA treated cats. The histogram compares averaged passing times between normal and IAA treated animals. The vertical coordinate depicts averaged passing time for normal $(7.21 \pm 2.55)$ and IAA treated $(57.33 \pm 4.62)$ animals $(P<0.01, t$-test $)$.

$(59.11 \pm 2.66, n=6)$ and always run-off obstacles before exiting. Indeed, Figure 5 clearly demonstrates that IAA treated animals completely lost visual detection capability and were unable to navigate barricades. Together, evidence from this experiment confirms the two prior test results and reveals that the IAA treated animals completely lost visual function.

\subsection{Histochemical examination}

Finally, we evaluated histochemical characteristics of retinal structures in both normal and IAA treated animals. As shown in Figure 6, in comparison with normal retina (Figure $6 \mathrm{~B}$ ), the ONL had virtually disappeared at all locations from center to peripheral retina in the IAA treated animal (Figure $6 \mathrm{~A}$ and $\mathrm{C}-\mathrm{E}$ ). It is evident that GCL of the IAA treated retina appears spared from the IAA induced damages.

\section{Discussion}

The present study demonstrates that IAA can be used to induce a complete elimination of photoreceptors and results in thorough abolition of ERG responses. Consequently, behavioral test confirms that IAA treated animal entirely lost visual function. Together, we present evidence that the IAA treatment produced an acute cat model of complete blindness that can be used for evaluation of visual function restoration or related experiments. Genetically modified feline model of photoreceptor degeneration has been developed for over the years [16-21]. However, one of the disadvantages of these animal models is the slow progress of the photoreceptor degeneration. For instance, it could be developed over the period of $12-16$ months $[18,19]$ or up to four years $[16,17]$. Furthermore, one of the most inevitable drawbacks is the unpredictable disease status of the animal model. For instance, one could not predict the degree of blindness in photoreceptor depleting animals. To the extreme, there is no animal model available for acute complete photoreceptor depletion. Thus, the acute and complete photoreceptor degeneration reported here could offer a valuable animal model for biological interventions such as gene therapy at the end stage of retinal disease.

The IAA has been extensively used as an effective tool to selectively eliminate retinal photoreceptors in a variety of species for the last 60 years $[1,3,4,10,11,14,22]$. As observed in other species, the toxic effects of IAA treatment are dose dependent. Noell noted that a single injection of the toxin through a limb vein $\left(12 \mathrm{mg} \mathrm{kg}^{-1}\right)$ induced acute optic nerve potential and ERG amplitude declinations simultaneously within $90 \mathrm{~s}$ after the injection and disappeared within the next minute [3]. However, the IAA abolished the ERG and other manifestations of the photoreceptor function

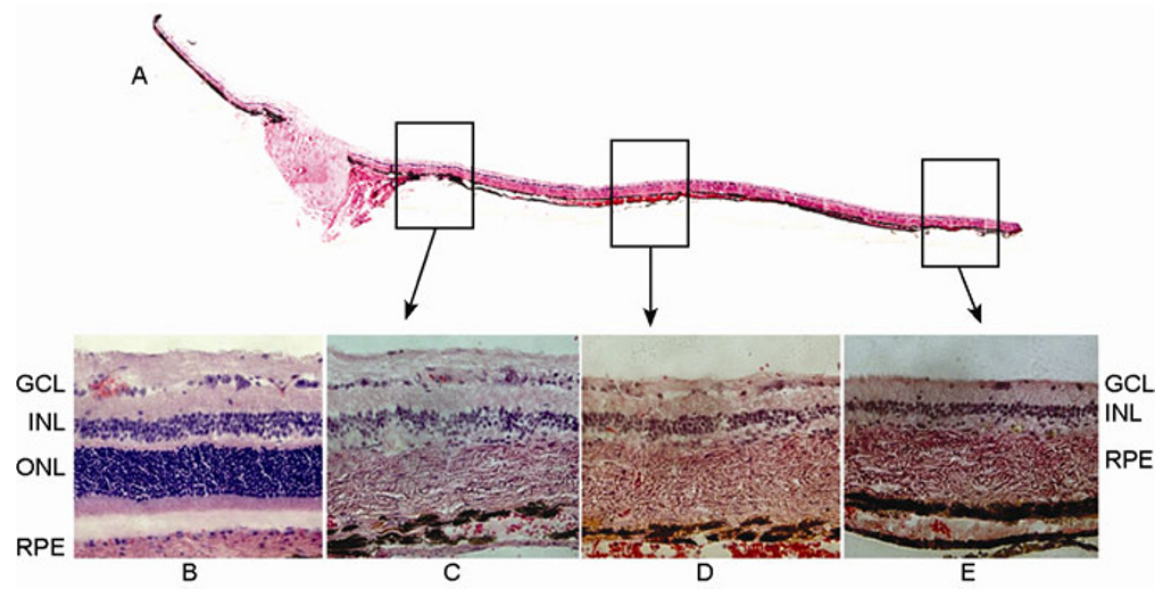

Figure 6 Sections of normal and IAA-treated feline retinas. A standard hematoxylin-eosin-saffranin protocol stained retinal section extending from the optic disc to the periphery of the retina three months after $20 \mathrm{mg} \mathrm{kg}^{-1}$ IAA treatment. A, An IAA treated cat retinal section. B, Normal retina section. ONL, outer nuclear layer; INL, inner nuclear layer; GCL, ganglion cell layer; RPE, retinal pigment epithelium. C-E, Retinal sections from different regions of the IAA treated retina (A). ONL had virtually disappeared at all locations from center part to periphery in IAA-treated retina. The remaining retinal layers appeared normal and the INL and GCL are readily discernible. 
only temporarily. The irreversible effects were produced only if the injection was repeated once or twice. Furthermore, it was also reported that, if the dose of a single injection increased to $22 \mathrm{mg} \mathrm{kg}^{-1}$, cats were found blind six weeks later and pathological examinations showed almost complete loss of outer and inner segments, all outer nuclei being pyknotic [4]. Thus higher dose of IAA should be pursued if the purpose is to induce complete blindness in cats. However, our pilot study showed that the tolerance to IAA varied substantially among cats and the dosage over the range of $21-25 \mathrm{mg} \mathrm{kg}^{-1}$ was lethal if the injection was made via a limb vein. Subsequently, Burke and Hayhow also made similar observations [23]. Alternatively by delivery IAA through an ocular artery, the long posterior ciliary arteries (LPCA) that provide blood supply to both the retina and choroid, Berson reported $30 \%-40 \%$ photoreceptors depletion with $0.268 \mathrm{mg} / 0.1 \mathrm{~mL}$ of IAA injection while the percentage increased to $50 \%$ if the dose was doubled $(0.52$ $\mathrm{mg} / 0.2 \mathrm{~mL}$ ) [21]. However, this method is technically challenging and the dosage of IAA for complete rods and cones removal remains to be determined. It was reported that, after a swine received a single injection of IAA via ear vein $\left(20 \mathrm{mg} \mathrm{kg}^{-1}\right.$ ), at two weeks post-IAA there was no rod- or cone-driven ERG response [11]. Thus, it is plausible that ear vein injection is more effective than limb vessel delivery. Indeed, after we adopted this alternative approach the successful rate was greatly improved. However, we noted that animals temporarily lost visual function during the first week of the IAA treatment, but some of them gradually regained partial visual functions during several weeks. Nevertheless, it was observed that if an animal is blinded for more than three months, few regained any visual functions. Thus by using this technique we successfully produced IAA induced acute blindness in cats.

A considerable drawback of high dosage IAA treatment is the adverse effects of the toxin on animals. It was reported that malate could protect the life of an experimental animal by providing it with some suitable energy-producing intermediate which could bypass the iodoacetate block without affecting glycolysis. Indeed, it was demonstrated in rats [2]. Unfortunately, we tried this approach on four cats but failed. It could be a species difference. However, compensating for the adverse effects of IAA should be a subject of future investigation.

It was observed in the present experiment that complete elimination of photoreceptors did not result in complete abolition of PLR. It is expected because light signal is sensed by two different types of photoreceptors, the conventional photoreceptors and intrinsically photosensitive retinal ganglion cells or melanopsin expressing retinal ganglion cells (ipRGCs), and the latter is much less sensitive than conventional photoreceptors [25]. Although there is substantial evidence that both conventional photoreceptors and ipRGCs contribute to PLR, depleting ipRGCs could substantially diminish PLR [25-27]. Since there is no mel- anopsin antibody available that specifically targets ipRGCs in the cat, we cannot evaluate the impact of IAA on ipRGCs in the cats. Nevertheless, the present result reveals that complete removal of conventional photoreceptors substantially reduced but did not completely abolish PLR. Thus our result is in agreement with previous reports [25-27] and suggests that the residual PLR could be contributed by surviving ipRGCs.

We performed behavioral test to determine if the animals had any residual visual functions. As shown in Figure 5, the blinded cats showed significantly slower passing time than the controls. However, the behavioral activity patterns varied among the blinded animals. We realized that one cannot identify a blinded animal only relying on number of obstacles the animal run-off during the test. For instance, one blind cat did not knock over any obstacles but the activity pattern of the animal showed that it did not visually identify any of those obstacles but sensed the objects with its whiskers or other body parts such as paws. On the other hand, one cannot judge if an animal is blind by solely relying on the passing time. We encountered one of the blinded animals that had short running time as controls but it knocked over all obstacles on the way to the end of the test course. The animal was calm during the test and its behavioral pattern showed that it did not recognize any of the obstacles before bumping into it. Thus, we did not include this animal's running time in Figure 5.

In conlusion, high dose IAA application $\left(20 \mathrm{mg} \mathrm{kg}^{-1}\right)$ via ear vein injection created an acute and reliable complete photoreceptor degeneration model in the cat. This model can be applied to genetic and cellular therapies for visual function restoration.

This work was supported by National Basic Research Program of China (2009CB320900 and 2011CB510206 to Pu MingLiang, 2011CB510200 to Li XiaoXin) and National Natural Science Foundation of China (30831160516 to Pu MingLiang).

1 Winkler B S, Sauer M W, Starnes C A. Modulation of the Pasteur effect in retinal cells: implications for understanding compensatory metabolic mechanisms. Exp Eye Res, 2003, 76: 715-723

2 Graymore C, Tansley K. Iodoacetate poisoning of the rat retina. I. Production of retinal degeneration. Br J Ophthalmol, 1959, 43: 177185

3 Noell W K. The effect of iodoacetate on the vertebrate retina. J Cell Comp Physiol, 1951, 37: 283-282

4 Noell W K. The impairment of visual cell structure by Iodoacetate. J Cell Comp Physiol, 1952, 40: 25-55

5 Noell W K. Studies on the electrophysiology and the metabolism of the retina. U.S.A.F. School of Aviation Medicine, Project No. 21-1201-0004, Report 1, Randolph Field, Texas, 1953. 1-122

6 Orzalesi N, Calabria G A, Grignolo A. Experimental degeneration of the rabbit retina induced by iodoacetic acid. A study of the ultrastructure, the rhodopsin cycle and the uptake of 14C-labeled iodoacetic acid. Exp Eye Res, 1970, 9: 246-253

7 Liang L, Katagiri Y, Franco L M, et al. Long-term cellular and regional specificity of the photoreceptor toxin, iodoacetic acid (IAA), in the rabbit retina. Vis Neurosci, 2008, 25: 167-177 
8 Yamauchi Y, Agawa T, Tsukahara R, et al. Correlation between high-resolution optical coherence tomography (OCT) images and histopathology in an iodoacetic acid-induced model of retinal degeneration in rabbits. Br J Ophthalmol, 2011, 95: 1157-1160

9 Farber D B, Souza D W, Chase D G. Cone visual cell degeneration in ground squirrel retina: disruption of morphology and cyclic nucleotide metabolism by iodoacetic acid. Invest Ophthalmol Vis Sci, 1983, 24: 1236-1249

10 Scott P A, Kaplan H J, Sandell J H. Anatomical evidence of photoreceptor degeneration induced by iodoacetic acid in the porcine eye. Exp Eye Res, 2011, 93: 513-527

11 Noel J M, Fernandez de Castro J P, Demarco P J Jr, et al. Iodoacetic acid, but not sodium iodate, creates an inducible swine model of photoreceptor damage. Exp Eye Res, 2012, 97: 137-147

12 Yamamoto F, Honda Y. Effects of intravenous iodoacetate and iodate on $\mathrm{pH}$ outside rod photoreceptors in the cat retina. Invest Ophthalmol Vis Sci, 1993, 34: 2009-2017

13 Winkler B S, Sauer M W, Starnes C A. Modulation of the Pasteur effect in retinal cells: implications for understanding compensatory metabolic mechanisms. Exp Eye Res, 2003, 76: 715-723

14 Wang W, Fernandez de Castro J, Vukmanic E, et al. Selective rod degeneration and partial cone inactivation characterize an iodoacetic acid model of Swine retinal degeneration. Invest Ophthalmol Vis Sci, 2011, 52: 7917-7923

15 Nan Y, Xiao C, Chen B, et al. Visual response properties of Y cells in the detached feline retina. Invest Ophthalmol Vis Sci, 2010, 51: 1208-1215

16 Narfström K. Progressive retinal atrophy in the Abyssinian cat. Sven
Vet Tidn, 1981, 33: 147-150

17 Narfström K. Hereditary progressive retinal atrophy in the Abyssinian cat. J Hered, 1983, 74: 273-276

18 Barnett K C, Curtis R. Autosomal dominant progressive retinal atrophy in Abyssinian cats. J Hered, 1985, 76: 168-170

19 Curtis R, Barnett K C, Leon A. An early-onset retinal dystrophy with dominant inheritance in the Abyssinian cat. Clinical and pathological findings. Invest Ophthalmol Vis Sci, 1987, 28: 131-139

20 Rah H, Maggs D J, Blankenship T N, et al. Early-onset, autosomal recessive, progressive retinal atrophy in Persian cats. Invest Ophthalmol Vis Sci, 2005, 46: 1742-1747

21 Menotti-Raymond M, Deckman K H, David V, et al. Mutation discovered in a feline model of human congenital retinal blinding disease. Invest Ophthalmol Vis Sci, 2010, 51: 2852-2859

22 Berson E L. Acute toxic effects of chloroquine on the cat retina. Invest Ophthalmol, 1970, 9: 618-628

23 Burke W, Hayhow W R. Disuse in the lateral geniculate nucleus of the cat. J Physiol, 1968, 194: 495-519

24 Berson D M, Dunn F A, Takao M. Phototransduction by retinal ganglion cells that set the circadian clock. Science, 2002, 295: 1070-1073

25 Lucas R J, Hattar S, Takao M, et al. Diminished pupillary light reflex at high irradiances in melanopsin-knockout mice. Science, 2003, 299: 245-247

26 Güler A D, Ecker J L, Lall G S, et al. Melanopsin cells are the principal conduits for rod-cone input to non-image-forming vision. Nature, 2008, 453: 102-105

27 Do M T, Kang S H, Xue T, et al. Photon capture and signalling by melanopsin retinal ganglion cells. Nature, 2009, 457: 281-287

Open Access This article is distributed under the terms of the Creative Commons Attribution License which permits any use, distribution, and reproduction in any medium, provided the original author(s) and source are credited. 